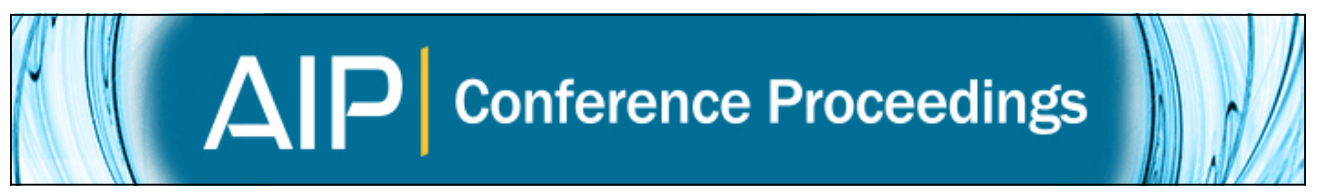

\title{
Spatially Resolved Chandra HETG Spectroscopy of the NLR Ionization Cone in NGC 1068
}

D. A. Evans, P. M. Ogle, H. L. Marshall, M. A. Nowak, S. Bianchi, M. Guainazzi, D. Dewey, N. S. Schulz, M. S. Noble, J. Houck, and C. R. Canizares

Citation: AIP Conference Proceedings 1248, 425 (2010); doi: 10.1063/1.3475282

View online: http://dx.doi.org/10.1063/1.3475282

View Table of Contents:

http://scitation.aip.org/content/aip/proceeding/aipcp/1248?ver=pdfcov

Published by the AIP Publishing

\section{Articles you may be interested in}

Chandra High Resolution Imaging of NGC 1365 and NGC 4151

AIP Conf. Proc. 1248, 519 (2010); 10.1063/1.3475330

High resolution spectroscopy as a tool to study line emitting material in AGNs

AIP Conf. Proc. 1248, 485 (2010); 10.1063/1.3475312

Suzaku and XMM-Newton Observations of Type 2 ULIRGs with High [OIII] Luminosity AIP Conf. Proc. 1248, 445 (2010); 10.1063/1.3475291

Thermal Instabilities in the Wind of NGC 3783

AIP Conf. Proc. 1248, 439 (2010); 10.1063/1.3475288

Nature of the soft X-ray emission in LINERs through RGS/XMM-Newton spectra AIP Conf. Proc. 1248, 343 (2010); 10.1063/1.3475254 


\title{
Spatially Resolved Chandra HETG Spectroscopy of the NLR Ionization Cone in NGC 1068
}

\author{
D. A. Evans*, P. M. Ogle ${ }^{\dagger}$, H. L. Marshall*, M. A. Nowak*, S. Bianchi**, \\ M. Guainazzi ${ }^{\star}$ D. Dewey*, N. S. Schulz*, M. S. Noble* J. Houck* and \\ C. R. Canizares* \\ ${ }^{*}$ MIT Kavli Institute for Astrophysics and Space Research, 77 Massachusetts Avenue, Cambridge, \\ MA 02139, USA \\ ${ }^{\dagger}$ Spitzer Science Center, California Institute of Technology, Mail Code 220-6, Pasadena, CA \\ 91125, USA \\ ** Dipartimento di Fisica, Universita degli Studi Roma Tre, via della Vasca Navale 84, I-00146 \\ Roma, Italy \\ ${ }^{\ddagger}$ European Space Astronomy Centre of ESA, P.O.Box 78, Villanueva de la Canada, E-28691 \\ Madrid, Spain
}

\begin{abstract}
We present initial results from a new 440-ks Chandra HETG GTO observation of the canonical Seyfert 2 galaxy NGC 1068. The proximity of NGC 1068, together with Chandra's superb spatial and spectral resolution, allow an unprecedented view of its nucleus and circumnuclear NLR. We perform the first spatially resolved high-resolution X-ray spectroscopy of the 'ionization cone' in any AGN, and use the sensitive line diagnostics offered by the HETG to measure the ionization state, density, and temperature at discrete points along the ionized NLR. We argue that the NLR takes the form of outflowing photoionized gas, rather than gas that has been collisionally ionized by the small-scale radio jet in NGC 1068. We investigate evidence for any velocity gradients in the outflow, and describe our next steps in modeling the spatially resolved spectra as a function of distance from the nucleus.
\end{abstract}

Keywords: galaxies: active - galaxies: individual (NGC 1068) - X-rays: galaxies

PACS: $98.54 .-\mathrm{h}$, 98.62.Js

\section{OVERVIEW: THE ROLE OF AGN OUTFLOWS IN GALAXY EVOLUTION}

Outflows and feedback from AGN are widely invoked as the key mediator between the co-evolution of black holes and their host galaxies over cosmic time. It is now well understood from large optical surveys that galaxies evolve through mergers from blue, star-forming spirals (the 'blue cloud') whose black holes accrete at or close to their Eddington limits, through a transition region (the 'green valley'), to so-called 'red and dead' ellipticals (the 'red sequence'), which instead are described by markedly less black-hole growth. The importance of outflows in this evolution has now taken center stage; yet before we can successfully incorporate AGN feedback into numerical simulations of galaxy growth, a number of key questions need to be answered from observations:

- Can AGN actually deliver enough power to their environments to alter the evolution of their host galaxies in a meaningful way? 

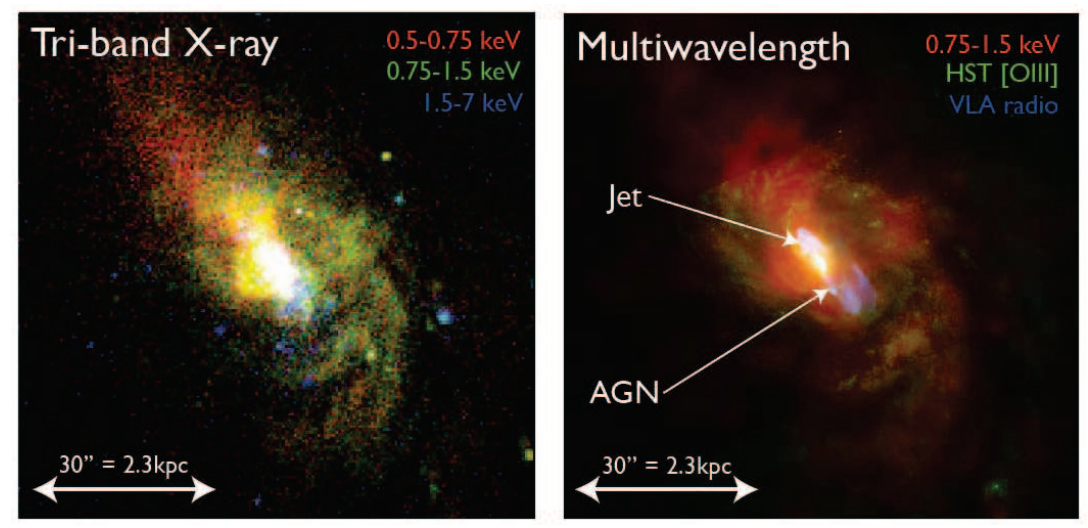

FIGURE 1. (left): HETG zero-order image of NGC 1068. Shown are events in the $0.5-0.75 \mathrm{keV}$ (red), 0.75-1.5 keV (green), and 1.5-7 keV (blue) energy bands. (right): Multiwavelength composite of NGC 1068, in 0.75-1.5 keV X-rays (red), HST [O III] (green), and VLA radio (blue) bands.

- On what spatial scales does this occur?

- Where are the outflows in the first place?

\section{X-RAY OBSERVATIONS OF OUTFLOWS IN AGN: INTRODUCING NGC 1068}

Observations of Type 1 Seyfert galaxies with the high-resolution X-ray gratings instruments on board Chandra and XMM-Newton have yielded strong evidence for outflows seen in absorption against the nuclear continuum, with velocities of hundreds to thousands of $\mathrm{km} \mathrm{s}^{-1}$ (e.g., [8, 9]). However, owing to the face-on orientation of these systems with respect to the observer, there is little information on the spatial extent of these outflows. The kpc-scale NLR in Type 2 Seyfert galaxies, on the other hand, which have edge-on inclinations, do not suffer from this orientation issue; yet since only a small percentage of the nuclear flux is scattered into the line of sight by a medium external to the AGN, the signal-to-noise of any outflow seen this time in emission tends to be poor and is dominated by the nucleus, rather than the off-nuclear gas.

There is one AGN, the prototypical Seyfert 2 galaxy NGC 1068, that is sufficiently near $\left(z=0.003793 ; 1^{\prime \prime}=80 \mathrm{pc}\right)$ and bright that we can, in principle, perform spatially resolved, high-resolution gratings spectroscopy of both the nucleus and any off-nuclear gas. NGC 1068 has a $10^{7} M_{\odot}$ black hole that is accreting at or close to its Eddington limit (e.g., [5]), making it an excellent source to examine the role of AGN outflows on galaxy-scale gas on a galaxy in the 'green valley'. NGC 1068 has been extensively studied at optical wavelengths; spatially resolved HST spectroscopy [1] shows that the NLR takes the form of a series of radiatively driven outflowing clouds, but its distribution is complex and cannot be modeled by a simple biconical outflow. NGC 1068 also shows evidence of a kpc-scale radio jet [11], meaning that we can investigate the competing 
roles of collisional ionization from the radio ejecta and photoionization from the AGN radiation field. We can also compare these effects between Seyfert galaxies and radioloud AGN, such as the well-studied radio galaxy $3 \mathrm{C} 33[6,10]$.

We have recently obtained a 440-ks Chandra HETG GTO observation of NGC 1068 (PI Canizares), and present our initial results here. In particular, we use:

1. Multiwavelength imaging of kpc-scale circumnuclear gas; and

2. High-resolution Chandra HETG spectroscopy

to study the NLR in NGC 1068, and in doing so determine the physical conditions and kinematics of any AGN-induced outflow.

\section{MULTIWAVELENGTH IMAGING OF NGC 1068}

In Figure 1, we show a tri-color X-ray image of NGC 1068 (left), together with a multiwavelength (X-ray, [O III] and radio) overlay (right). It is clear from the left panel that the hard X-ray emission tends to principally be concentrated in the nucleus itself, though there is evidence that it extends $\sim 1 \mathrm{kpc}$ from the AGN in a biconical distribution. The soft X-rays are much more spatially elongated, and extend several kpc from the nucleus in either direction. The multiwavelength image (right panel) demonstrates the complex morphology between the X-ray and [O III] NLR, and the kpc-scale radio jet. Although there is evidence for jet-gas interactions, it is evident that the soft X-ray emission and [O III] lie several kpc beyond the radio ejecta, which suggests that shockheating of gas by the jet does not dominate the energetics of the source. However, it is only by performing high-resolution spectroscopy that we can distinguish between collisional ionization from the jet and photoionization from the AGN radiation field, and test if the extended X-ray gas is ambient or outflowing.

\section{HETG SPECTROSCOPY OF NGC 1068}

In Figure 2 we show the co-added Chandra MEG and HEG spectrum of the nucleus of NGC 1068 from the entire 440-ks observation. We plot the principal transitions of $\mathrm{H}$ and He-like species, together with radiative recombination continuua (RRCs). Many of these transitions have already been identified by [4] and [7]. We detect clear evidence for blueshifts in the lines, which indicate that the gas is outflowing, with velocities of several hundred $\mathrm{km} \mathrm{s}^{-1}$. The detection of narrow RRCs and the ratios of the resonance, intercombination, and forbidden lines strongly suggest that the gas is photoionized by the AGN radiation field and takes the form of an outflow.

\section{NEXT STEPS: SPATIALLY RESOLVED HETG SPECTROSCOPY}

There is sufficient signal-to-noise to enable us to perform detailed photoionization modeling of the off-nuclear gas along this ionization cone. Our preliminary work indicates that outflows of several hundred $\mathrm{km} \mathrm{s}^{-1}$ are detected out to $1 \mathrm{kpc}$ from the AGN. Our 

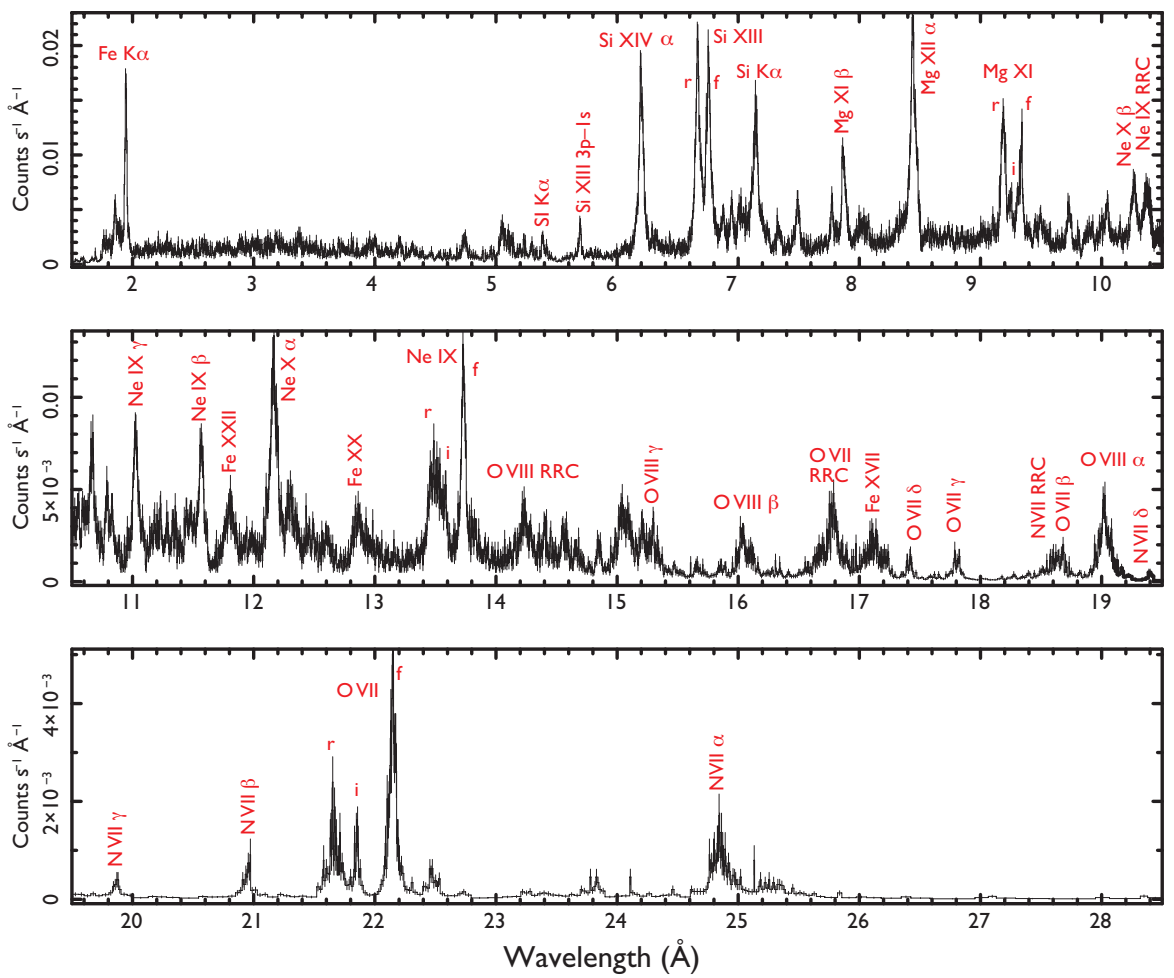

FIGURE 2. Co-added Chandra HETG MEG and HEG gratings spectrum of NGC 1068 from the entire 440-ks data set. Shown are the principal transitions from H- and He-like species, together with RRCs.

photoionization calculations are ongoing, but we will be soon able to calculate the mass outflow rate and power deposited by the photoionized outflow into the galaxy-scale gas [2], providing key constraints need to answer the long standing question about the role of AGN outflows on galaxy evolution.

\section{REFERENCES}

1. Das, V., Crenshaw, D. M., \& Kraemer, S. B. 2007, ApJ, 656, 699

2. Evans, D. A., et al. 2010, in prep.

3. Hickox, R. C., et al. 2009, ApJ, 696, 891

4. Kinkhabwala, A., et al. 2002, ApJ, 575, 732

5. Kishimoto, M. 1999, Advances in Space Research, 23, 899

6. Kraft, R. P., et al. 2007, ApJ, 659, 1008

7. Ogle, P. M., Brookings, T., Canizares, C. R., Lee, J. C., \& Marshall, H. L. 2003 A\&A, 402, 849

8. Reeves, J. N., Sambruna, R. M., Braito, V., \& Eracleous, M. 2009, ApJL, 702, L187

9. Steenbrugge, K. C., Fenovčík, M., Kaastra, J. S., Costantini, E., \& Verbunt, F. 2009, A\&A, 496, 107

10. Torresi, E., et al. 2009, A\&A, 498, 61

11. van der Hulst, J. M., Hummel, E., \& Dickey, J. M. 1982, ApJL, 261, L59 